\title{
Nach der Akkreditierung ist vor der Akkreditierung
}

Wie jedes Jahr bietet der Geschäftsbericht die Gelegenheit, auf der Basis von Kennzahlen und Fakten über die Aktivitäten des SIWF und über besondere Themen zu berichten, welche die Mitarbeitenden, die Geschäftsleitung und den Vorstand beschäftigt haben.

Ein markantes Ereignis für das SIWF und für die Fachgesellschaften war der erfolgreiche Abschluss der Akkreditierung durch das Eidgenössische Departement des Innern. Diese bestätigt, dass alle Weiterbildungsgänge wiederum für sieben Jahre anerkannt sind und das SIWF weiterhin im staatlichen Auftrag die Weiterbildungsstätten anerkennen und die eidgenössischen Facharzttitel verleihen kann. Dies bedeutet aber nicht: "Zurücklehnen und warten auf die Akkreditierung 2025». Gerade in unserer Zeit der schnellen Veränderungen von Strukturen und Rahmenbedingungen im Gesundheitswesen sind wir dafür verantwortlich, die Anforderungen an die Weiterbildung laufend zu überprüfen und zu aktualisieren. Ein Beispiel dafür sind die Auswahl und die Anzahl bestimmter Interventionen, die in den Lernzielkatalogen gefordert werden. Auch unser Engagement für genügende Ressourcen, auf welche die Weiterbildung der nächsten Ärztegeneration angewiesen ist, bleibt dringend notwendig.

Der Alltag im SIWF ist geprägt durch die möglichst speditive, aber sorgfältige Bearbeitung der grossen Zahl an Gesuchen um Verleihung eines Facharzttitels oder eines Schwerpunktes und um die Anerkennung von Weiterbildungsstätten. Neben anderen Arbeitsbereichen, wie der Vergabe von Fortbildungs-Credits, der Organisation von Visitationen und von Tagungen zur ärztlichen Bildung, der Erteilung unzähliger Auskünfte sowie der Ausschreibung des SIWF-Awards fordert uns immer noch ganz besonders die Neuentwicklung des e-Logbuchs, ein Grossprojekt, das aber planmässig vorangeht.

Zur Erfüllung aller Aufgaben des SIWF tragen die Mitarbeitenden, die Vorstands- und Kommissionsmitglieder das Entscheidende bei. Ihnen danken wir ganz herzlich für ihr Engagement und für ihre Unterstützung.

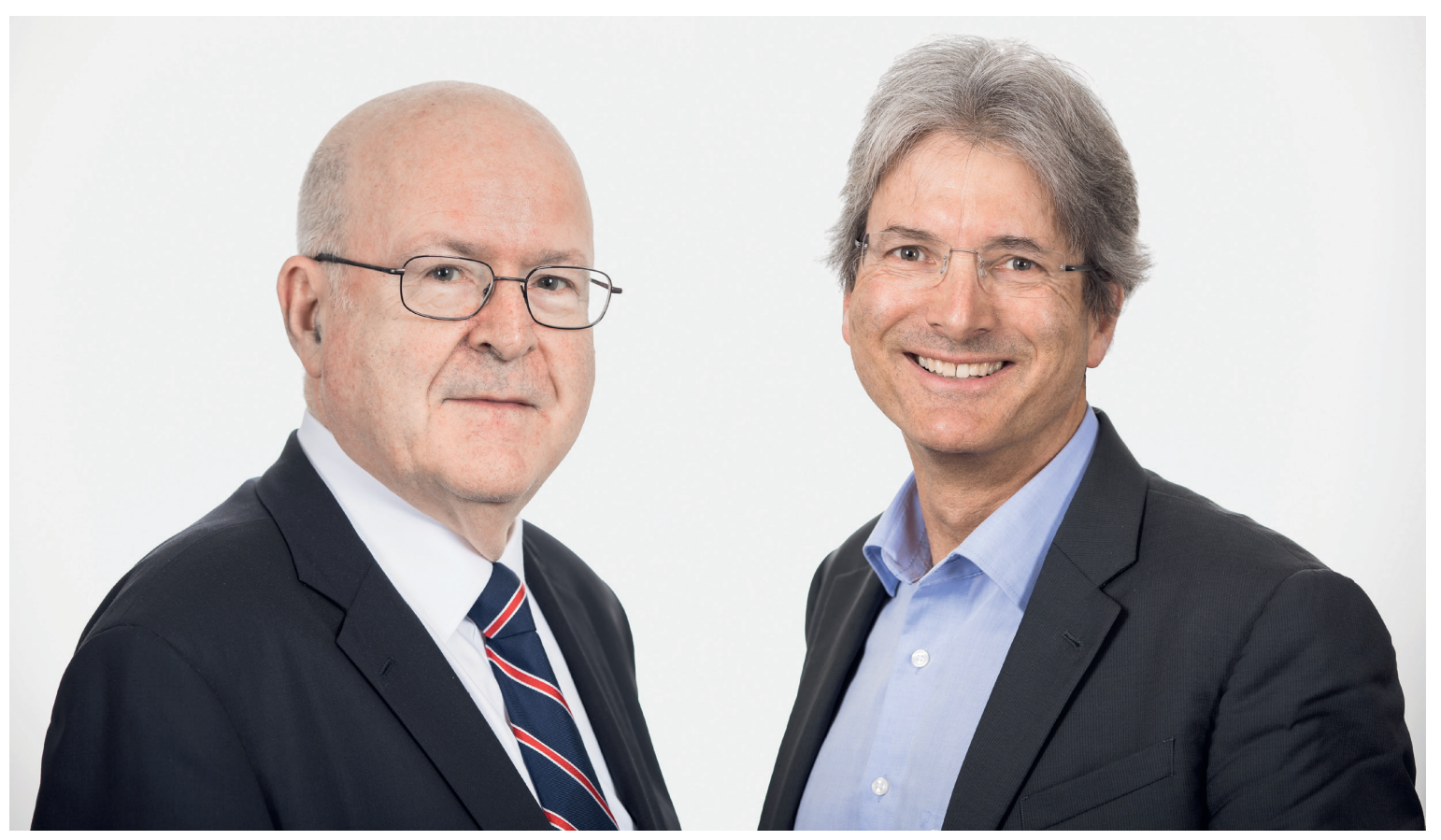

Dr. med. Werner Bauer, Präsident SIWF, Christoph Hänggeli, Rechtsanwalt, Geschäftsführer SIWF. 


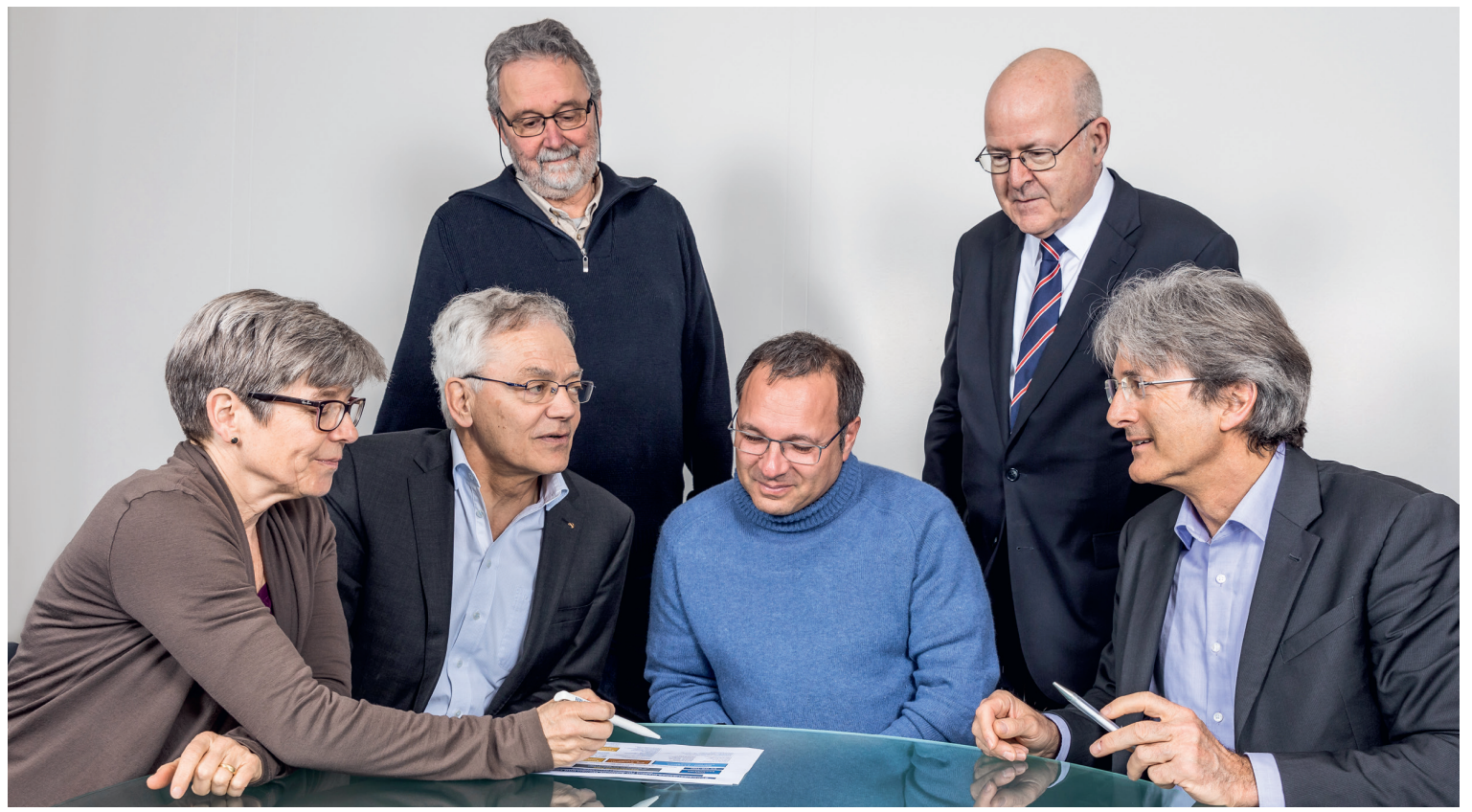

Die Geschäftsleitung des SIWF: Regula Schmid, Giatgen A. Spinas, Jean Pierre Keller, Raphael Stolz, Werner Bauer und Christoph Hänggeli (v.l.n.r.).

\section{Für sieben Jahre akkreditiert: Die Weiterbildung erhält gute Noten}

\section{Diplomaten benötigen in ihrem jeweiligen Gastland eine Akkreditierung. Was bedeutet dieser Begriff im Zusammenhang mit der Weiterbildung?}

Christoph Hänggeli: In der Schweiz regelt das Medizinalberufegesetz die Weiterbildung zur Fachärztin oder zum Facharzt. Diese führt seit 2002 zu einem eidgenössischen Titel. Vorher war es die Verbindung der Schweizer Ärztinnen und Ärzte (FMH), die als private Organisation die Spezialarztdiplome vergab, damals «FMH-Titel» genannt. Gemäss Gesetz ist im Auftrag des Bundes eine einzige schweizerische Organisation für die Weiterbildung verantwortlich. Diese Organisation ist das SIWF. Alle sieben Jahre evaluiert das Eidgenössische Departement des Innern (EDI) die Weiterbildungsgänge und beurteilt, ob das SIWF und die Fachgesellschaften ihre Aufgabe genügend gut erfüllen, um für eine weitere Periode damit betraut zu werden.

\section{Das klingt nach einem anspruchsvollen Prozedere.} Wie läuft denn diese Akkreditierung ab? Werner Bauer: Tatsächlich ist der Aufwand für eine aus- sagekräftige Beurteilung beträchtlich. Miteinbezogen sind das Bundesamt für Gesundheit (BAG), die Agentur für Akkreditierung und Qualitätssicherung (AAQ), die Medizinalberufekommission des Bundes (MEBEKO), das SIWF und alle Fachgesellschaften mit Verantwortung für einen Facharzttitel. Der Weg führt von der Erarbeitung von Qualitätsstandards durch das BAG und die AAQ über das Verfassen eines ausführlichen Selbstevaluationsberichts durch das SIWF und durch jede Fachgesellschaft zu den «tables rondes». Das sind Hearings mit nationalen und internationalen Experten. Ihr Gutachten dient als Grundlage für den Akkreditierungsentscheid.

\section{Wer entscheidet letztlich über die Akkreditierung?}

Christoph Hänggeli: Die Fachgesellschaften und das SIWF können jeweils zu ihrem Gutachten Stellung nehmen. Als Nächstes bereitet das BAG den Entscheid vor. Dazu gehört eine Anhörung der Medizinalberufekommission. Den Entscheid schliesslich fällt der Vorsteher des EDI. Von seinem Schreibtisch erreichen uns rund 45 Verfügungen, denn jeder Weiterbildungsgang 
erhält seine eigene Akkreditierung. Den Dokumenten beigelegt ist jeweils ein Einzahlungsschein, werden uns doch die Kosten für das ganze Prozedere auferlegt, die insgesamt gegen eine Million Franken betragen. Man kann in guten Treuen verschiedener Meinung darüber sein, ob wir für die Erlaubnis zum Wahrnehmen einer Aufgabe, die für die Zukunft des Gesundheitswesens zentral ist, auch noch bezahlen müssen. Die Fachgesellschaften werden damit aber nicht belastet, die Rechnungen werden vom SIWF übernommen.

\section{Der Aufwand für die Akkreditierten dürfte auch - abgesehen von den Gebühren - hoch gewesen sein?} Raphael Stolz: Die zeitliche Belastung für die Verantwortlichen in den Fachgesellschaften und im SIWF war massiv. Der Selbstevaluationsbericht umfasste in neun Kapiteln eine Vielzahl von Einzelfragen zu allen denkbaren Qualitätsbereichen und zusätzlich eine Gesamtbeurteilung. Das Erstellen dieser Dokumente war zeitintensiv und hat immer wieder zu Seufzern bei einzelnen Verantwortlichen geführt, die sich ja im Milizsystem, das heisst meist in der Freizeit, für die Weiterbildung engagieren. Wir konnten trotzdem feststellen, dass die Qualität der Berichte und auch der Voten in den Expertengesprächen mit wenigen Ausnahmen erfreulich gut war und Dank und Respekt verdienen.

\section{Was war denn schliesslich das Ergebnis der Akkreditierung?}

Werner Bauer: Dazu möchte ich einen zentralen Satz von Bundesrat Alain Berset aus seinem Brief an das SIWF zitieren: «Es freut mich sehr, dass alle diese im Verfahren involvierten Akteure zu grundsätzlich positiven Einschätzungen der Qualität der Weiterbildungsgänge in Humanmedizin gekommen sind.» Sechs Fachgesellschaften haben eine Auflage bekommen, deren Bearbeitung verpflichtend ist. Daneben haben die Experten in den Gutachten verschiedenste Empfehlungen formuliert, die von den Fachgesellschaften bei zukünftigen Revisionen ihrer Weiterbildungsprogramme berücksichtigt werden können. Gewicht legt Bundesrat Berset auch auf die Autonomie des SIWF, schreibt er doch: «Dass das SIWF eine eigenständige, von Standesinteressen unabhängige Organisation ist, wird von der Gutachtergruppe als sinnvoll und notwendig beurteilt.»

\section{Also ein Happy End und sieben Jahre Ruhe?}

Giatgen Spinas: Wir dürfen mit dem Ergebnis der Ak- kreditierung sicher zufrieden sein und haben für weitere sieben Jahre das Mandat erhalten, eidgenössische Facharzttitel zu erteilen. Etwas bedauerlich, aber bei einem Akkreditierungsverfahren wohl nicht vermeidbar, ist die Tatsache, dass mit grossem Einsatz von Ressourcen der Ist-Zustand beschrieben und als anerkennungswürdig dargestellt werden muss, wogegen nach Herausforderungen oder auch Schwachpunkten zwar gefragt wird, diese aber nicht zu negativ ins Gewicht fallen dürfen. Dies bedeutet, dass sich die Fachgesellschaft und das SIWF nicht auf den "Akkreditierungslorbeeren» ausruhen dürfen, sondern rechtzeitig dafür zu sorgen haben, dass auch in sieben Jahren wieder ein erfreuliches Ergebnis resultiert. Die gegenwärtig stattfindenden Strukturveränderungen, die Entwicklungen bei den medizinischen und didaktischen Methoden, die bedrohten zeitlichen und finanziellen Ressourcen, die Konsequenzen der Digitalisierung und was der Herausforderungen mehr sind, machen ein entschlossenes Engagement notwendig, um das Niveau der Weiterbildung hoch zu halten.

\section{Sind beim Rückblick auf das Jahr 2018 weitere Themen erwähnenswert?}

Jean Pierre Keller: An weiteren Themen, die das SIWF beschäftigt haben, mangelt es nicht: Neben den alltäglichen Aufgaben wie Titelgesuche und Anträge von Weiterbildungsstätten beurteilen, über hundert Visitationen organisieren, die Fachgesellschaften bei der Revision von Weiterbildungsprogrammen unterstützen, die Fortbildungsplattform unterhalten und Credits für Fortbildungsveranstaltungen vergeben, ist vor allem der Neubau des e-Logbuchs arbeits- und kostenintensiv und hält uns in Atem.

Christoph Hänggeli: Das trifft zu. Wir haben ja erkennen müssen, dass das bisher verwendete Programm den Anforderungen nicht mehr gewachsen und nicht mehr ausbaubar ist. Das neue e-Logbuch ist für uns ein Grossprojekt. Wir gehen davon aus, dass die gestaffelte Inbetriebnahme 2019/2020 erfolgen kann.

Regula Schmid: Unbedingt zu erwähnen ist noch eine didaktische Methode, die für das Medizinstudium schon eingeführt wurde und nun auch bei der Weiterbildung an die Türe klopft. Es geht um die «entrustable professional activities» (EPA). Dabei geht man weg von den bisher üblichen Katalogen mit unzähligen Einzeller-Zielen hin zu Kompetenz-Sets, die schrittweise erworben und attestiert werden. Eine Arbeitsgruppe wird die Grundlagen für die Anwendung dieses innovativen Tools in der Weiterbildung erarbeiten.

Bildnachweis

Tobias Schmid/SIWF 


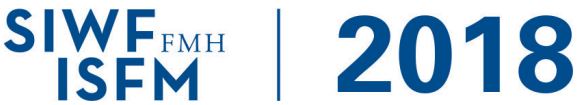

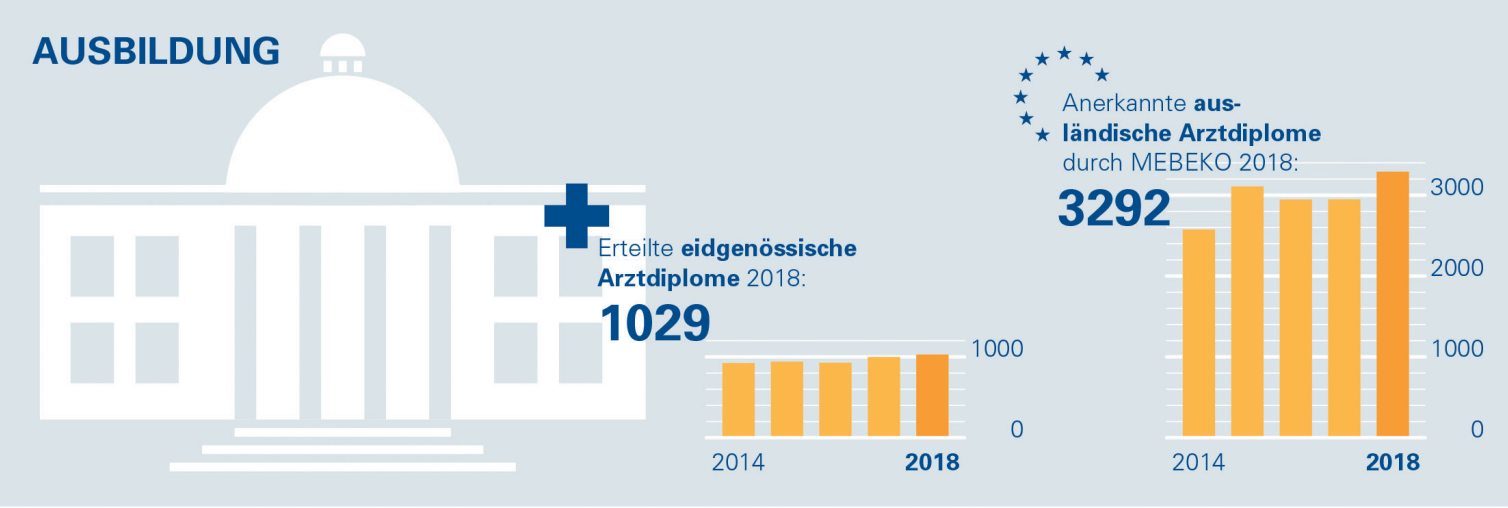

\section{WEITERBILDUNG}

e-Logbuch

Anzahl

registrierte

2018

2014

$18^{\prime} 000$

\section{FORTBILDUNG}

FORTBILDUNGSDIPLOME

Via e-Fortbildungsplattform Seit Inbetriebnahme selbst erfasste und von den der Fortbildungsplattform Fachgesellschaften gemeldete: 6144

$22^{\prime} 129$

Schwerpunkte Anzahl Facharzttitel und jeweilig 2018 erteilte:

Allgemeine Innere Medizin

Psychiatrie und Psychotherapie $117 / 8,7$ Jahre

Kinder- und Jugendmedizin

Durchschnittsalter Ärztinnen und

$\star^{\star} \stackrel{\star}{\star}$ Anerkannte ausländische

$\star$ Weiterbildungstitel durch MEBEKO 2018:

1392 2000

Ärte bei Ersterwerb Facharzttitel, alle: 36,2 Jahre

mit eidg. Diplom, $\leq 26$ Jahre und Weiterbildungsdauer $\leq 10$ Jahre: $\mathbf{3 2} \mathbf{8}$ Jahre

Fähigkeitsausweise 2018 erteilte:
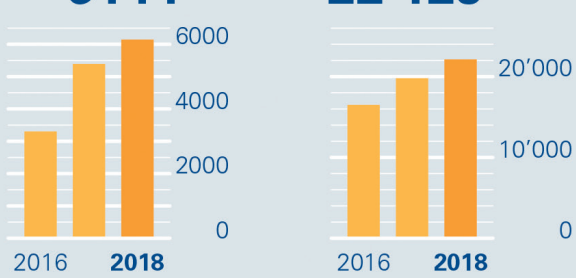

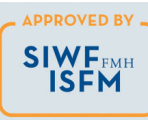

Anzahl vom SIWF anerkannte Fortbildungsveranstaltungen:

150

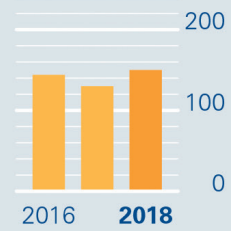




\section{$\underset{\substack{\text { SISFM } \\ \text { ISF }}}{\operatorname{SIW}} \mid \mathbf{2 0 1 8}$}

\section{FACHARZTTITEL}

Total erteilte Facharzttitel: 1434

davon an Ärzte mit CH-Diplom

davon an Ärzte mit ausländischem Diplom (die 10 häufigsten Herkunftsländer)
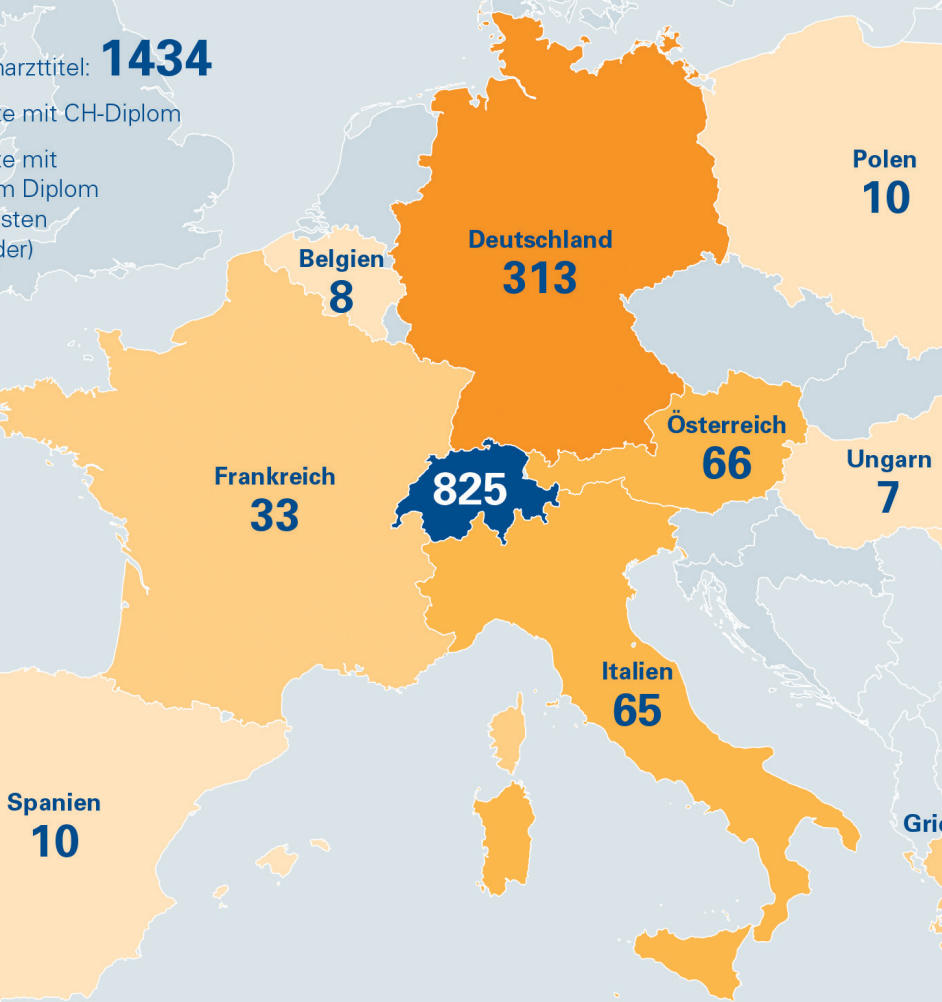

7

Rumänien

16

Griechenland

23

ASSISTENZ-

ÄRZTE-

UMFRAGE

\section{9}

Assistenzärztinnen und -ärzte

(Rücklaufquote $70,06 \%$ )

\section{8}

ausgewertete

Weiterbildungsstätten

79 von 100 Ärzten erachten den Beitrag der WBS zur Zusammenarbeit mit der Pflege als gross bis sehr gross.

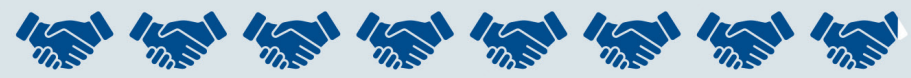

52 von 100 Ärzten erachten den ökonomischen Umgang

mit Ressourcen in Diagnostik und Therapie als wichtig bis sehr wichtig

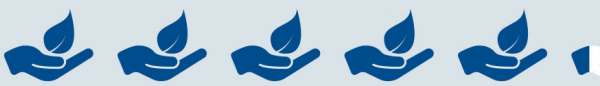

76 von 100 Ärzten würden die WBS weiterempfehlen.

it it ititititid 